\title{
Research on Marine sediment Measurement for the needs of Modern Marine Survey
}

\author{
Chao SUN ${ }^{1, *}$, Nan Wang ${ }^{1}$, Gang Bian ${ }^{1}$, Shaohua Jin ${ }^{1}$ \\ ${ }^{1}$ PLA Dalian Naval Academy Dalian, Liaoning,116000, China
}

\begin{abstract}
Marine sediment information is one of the important elements of marine spatial geographic information. Combined with the development and application requirements of marine geospatial information system, according to the requirements of marine sediment measurement and the characteristics of marine sediment information data, this paper comprehensively discusses three research problems: the application of acoustic instruments in marine sediment detection, the classification and expression of marine sediment measurement and the accuracy of interpolation methods.
\end{abstract}

\section{1 introduction}

Marine sediment is an important element on the chart and an important part of marine spatial geographic information. According to the marine sediment information data, it can deduce the trend of submarine ecological environment, marine dynamic environment and the development and change of the whole ocean. It can provide data support and decision-making basis for marine development and utilization, scientific research, marine engineering construction and maritime military and non-military activities. With the rapid development and progress of marine sediment measurement methods and technology, the problems such as the diversity of marine sediment data acquisition methods, data multi-sources, data complexity and the explosive growth of data are also the "happiness" troubles of modern scholars in the research or application of marine sediment. How to use multi-source marine sediment information data for research in order to have or even exceed the expected effect on users has been paid more and more attention by scholars at home and abroad. No matter how complex and diverse marine sediment information data are, the ultimate goal is to be expressed and used, so it requires scientific and complete classification and rich and intuitive expression of marine sediment data. Only in this way can we provide stronger support for the decision-making of marine information services ${ }^{[1]}$.

These studies are mainly focused on three aspects: the detection of marine sediment by acoustic instruments, the classification and expression of marine sediment information, and the interpolation method of marine sediment information.

\section{Application of Acoustic Instruments in Marine sediment Detection}

At present, the existing marine sediment detection methods can be divided into two categories: physical detection and acoustic instrument detection. Among them, physical detection is the earliest and most widely used method, that is, the treatment and analysis of marine sediment after physical sampling at the sediment detection station. However, this method also has some disadvantages, that is, the discontinuity of sampling points leads to discrete sediment data, and if the sampling points are encrypted, it will increase the economic cost and workload. In order to solve this kind of problem, on the basis of detecting seafloor topography with acoustic instruments, scholars continue to propose the feasibility of using acoustic instruments to detect and analyze marine sediment qualitatively[2].

In 2010, Giovanni et al. [3] used sounding data, scattering intensity data and angular response of scattering intensity to study the relationship between sediment particle size and seafloor seaweed distribution in scattering intensity. Using the angular response of scattering intensity, the contents of coarse particles of 1$16 \mathrm{~mm}$ and $0.016-0.5 \mathrm{~mm}$ in sediment and the relationship between them and scattering intensity were studied respectively. The relative scattering intensity thresholds of bottom sediments such as gravel, gravel sand and argillaceous sand with a small amount of gravel were also studied. In China, some experts and scholars have also carried out related research in the field of bottom sediments. In 2006, Lu Hailong et al.[4] used Bayesian method to classify the backscattering data of submarine multi-beam signals in Jiaozhou Bay, and achieved certain classification results, but the classification accuracy is not very high. In 2012, Zheng Hongbo et al. [5] used Biot

*Corresponding author: sunchao1990116@126.com 
theory to retrieve the porosity and permeability of the shallow sediment in a certain area of the northern South China Sea, so as to classify the sediment, but this inversion method is only suitable for the classification of soft sediment, but not suitable for the classification of hard sediment. In the face of such a variety of acoustic instruments and multi-source marine sediment information data, how to select these data to provide the basis for marine sediment information classification or which kind of data should be selected as the index elements of marine sediment classification needs to be further studied; secondly, whether the multi-source sediment information data can be fused or applied as a classification factor index. Whether data diversification can improve the quality control of marine sediment data measurement, improve detection accuracy, multi-data check analysis and so on.

\section{Classification and expression of Marine sediment Information}

In the aspect of marine sediment information classification, in 1954 Shepard [6] put forward a modern classification and nomenclature scheme of sediment by comparing and synthesizing several classification methods that existed at that time, which we called Shepard classification. It classifies and names the sediment types of the mixture according to the ratio of sand, silt and clay, and the three end members are completely equivalent and can be used for the general description and classification of sediment. In 1970, Folk [7] put forward a new classification scheme, namely Folk classification, based on the classification of gravel, sand, clay and silt. Folk classification is established on the basis of different ratios of sediment composition and energy. Gravel and non-gravel are expressed by two triangles respectively, in which each end element is not equivalent, which can scientifically reflect the marine dynamic conditions of sediment and then judge the sedimentary environment. In 2004, Goff et al studied the seabed characteristics of central New Jersey and the continental shelf, and obtained the correlation and spatial variability of seafloor sediment characteristics.

There are also many scholars studying the classification of marine sediment information in China. In 2000, Cheng Peng [8] sorted out and summarized the sedimentary and classification characteristics of particulate matter in the North Yellow Sea. In 2002, He Qixiang et al. [9] compared the classification schemes of marine sediment represented by Shepard and Folk, proposed that the classification of sediment should have both description and interpretation functions, and established a set of multiple classification system with matching structure classification and composition classification, which solved some problems in the classification of marine sediment, but still did not jump out of the classification scheme limited to grain size analysis. In 2012, Zhang Fuyuan et al [10] analyzed in detail the current situation of classification and nomenclature of deep-sea sediment at home and abroad, and put forward the classification and naming scheme of deep-sea sediment and its key technologies on the basis of improving their compatibility and comparability. For the classification scheme or system of marine sediment information, many literatures focus on the optimization and perfection of the classification scheme based on marine sediment grain size, especially the comparison and analysis of Shepard and Folk methods. Finally, a classification and naming scheme based on marine sediment particle size is proposed. In view of various marine sediment classification methods, some scholars have made a distinction between the deep sea and the shallow sea, and put forward different classification and naming schemes for marine sediment information for the deep sea or shallow sea, but so far there is no classification system considering multi-index classification elements, although a single classification standard or split index can initially complete the classification of marine sediment information. We can also get more complex classification items, but the final expression can not maximize the detection of the bottom information, can only show a certain type of index information, which is accepted by users or decision makers. According to the actual situation, the classification system of multi-index classification elements can systematically classify marine sediment information based on multi-index classification elements, and use all kinds of sediment information efficiently and fused.

\section{The problem of data interpolation method of Marine sediment Information}

In addition to the above two problems, the continuity of marine sediment site information under the classification system is also a common concern of scholars at home and abroad. Because now the most suitable and accurate method of marine sediment detection is the physical detection of sediment station sites, and these points are not continuous, how to make these points continuous is the study of the interpolation method of sediment information. At the beginning of the research, most of the sediment interpolation methods adopted by most scholars are based on the sediment granularity interpolation method, that is, under the triangulation structure, the particle size of a point in the region is obtained by surface interpolation composed of three known points, as shown in figure 1 and such as formula (1).

$$
\left\{\begin{array}{c}
Z_{p}=a_{0}+a_{1} x_{P}+a_{2} y_{P} \\
{\left[\begin{array}{l}
a_{0} \\
a_{1} \\
a_{2}
\end{array}\right]=\left[\begin{array}{lll}
1 & x_{A} & y_{A} \\
1 & x_{B} & y_{B} \\
1 & x_{C} & y_{C}
\end{array}\right]^{-1}\left[\begin{array}{l}
z_{A} \\
z_{B} \\
z_{C}
\end{array}\right]}
\end{array}\right.
$$

$\mathrm{Zp}$ is the average particle size of unknown point $\mathrm{P}$ (xp,yp) (xa,ya) (xb,yb) (xc,yc) is the geographic information of point $\mathrm{P}$, point $\mathrm{A}$, point $\mathrm{B}$ and point $\mathrm{C}$ respectively, and $\mathrm{a} 0, \mathrm{~A} 1$ and $\mathrm{a} 2$ are interpolation coefficients, respectively. 


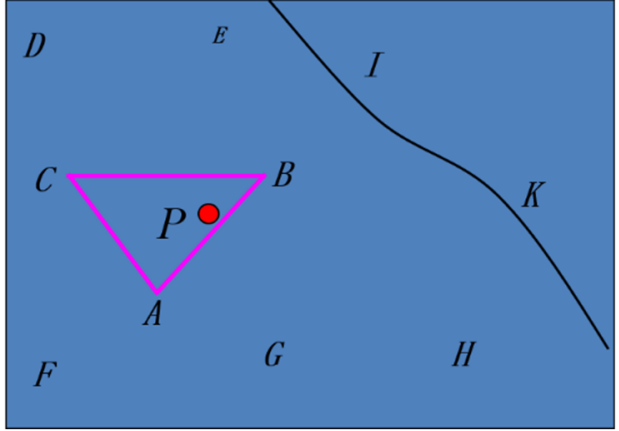

Fig 1 Construction of marine sediment granularity interpolation triangulation

At present, this kind of method is still widely used. Because of its simplicity, easy to understand and convenient operation, it can be used in the case of low precision and sufficient data information. But this method is based on the average grain size interpolation method, sometimes although the average grain size value is obtained, there is no in-depth understanding of the sediment information in the location area, and the characteristic parameters such as variance and median value are not available. It does not represent the sediment information in this area, and this method is not recommended when the accuracy is high or the change of sediment particle size in the test area is obvious. In 2011, Liu Fucheng et al. [11] put forward a non-parametric method for the production cost of offshore sediment type map. This method uses the sediment type map generation method indicating Kriging, which is more accurate than the traditional mapping method. In 2012, R.M.Lark et al.[12] proposed a marine sediment particle size interpolation method based on collaborative kriging, which tested the marine sediment particle size information in the experimental area, and obtained high precision results. In the study of the interpolation method of marine sediment information, the author noticed that scholars at home and abroad are bound by the classification system or the framework of classification ideas. Often only for the sediment grain size of a single sediment classification element to calculate to get the desired results. Kriging interpolation can be applied to marine sediment data processing. The normal distribution is shown in figure 2 .

Although such results have a certain effect on sediment particle size calculation, a single particle size parameter can not represent the visual expression of marine sediment information to the greatest extent, and such results may sometimes mislead users. It is difficult to make the right decision.

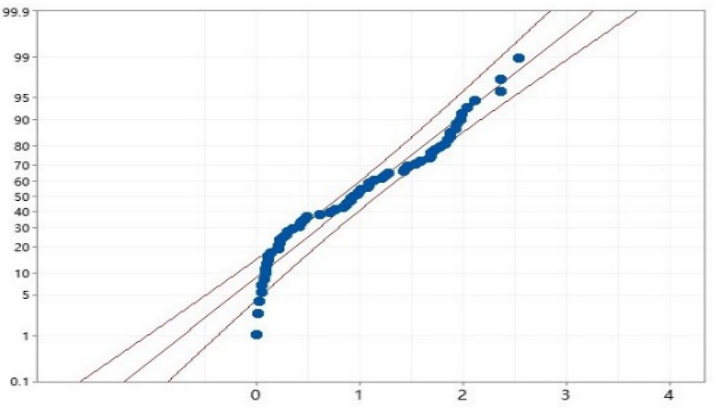

Fig 2 Normal probability map of sediment grain size in a sea area

\section{Summary}

To sum up, from the comprehensive analysis of the data at home and abroad, the marine sediment spatial data is an important part of marine spatial geographic information, although many researchers have begun to realize the importance of the research on the classification of marine sediment information. Some scholars have also begun to improve the shortcomings of classification methods, acoustic sediment detection instruments and other problems. However, for the establishment of a relatively perfect, multi-index sediment classification system and the needs of solving related problems, it has not yet formed a complete logical and systematic argumentation and analysis, and there is a lack of consideration for solving interpolation methods to meet the requirements of information expression. This paper makes a comprehensive discussion on the application of acoustic instruments in marine sediment detection, the classification and expression of marine sediment information and the interpolation of marine sediment information.

\section{References}

1 Li Gaixiao, Wang Mo, Cui Gaosong, et al. Research on Marine sediment Database engine Technology [J]. Marine surveying and Mapping, 2011 (04): 56-58.

2 Biot M A. Theory of propagation of elastic waves in a fluid-saturated porous solid : II. Higher frequency range $[\mathrm{J}]$. The Journal of the Acoustical Society of America, 1956, 28.

3 Ferrini V L, Flood R D . The effects of fine-scale surface roughness and grain size on 300

a) Khz multibeam backscatter intensity in sandy marine sedimentary environments[J]. Marine Geology, 2006, 228(1-4):153-172.

4 LV Hailong, du Dewen, Liu Yiguang, et al. Statistics of multi-beam echo data and application of sediment classification [J]. Advances in Marine Science, 2006,24 (004): 61-69.

5 Zheng H B, Yan P, Chen J, et al. Seabed sediment classification in the northern South China Sea using inversion method[J]. Applied Ocean Research, 2013, 39:131-136.

6 Shepard F P. Nomenclature Based on Sand-Silt-Clay Ratios [J]. Journal of sedimentary Petrology, 1954, 24(3):151-158.

7 Folk R L , Andrews P B, Lewis D W. Detrital sedimentary rock classificati on and nomenclature for use in New Zealand [J] . New Zealand Journal of Geology and Geophysics, 1970, 13(4): 937-968.

8 Cheng Peng. Sedimentary characteristics and transport process of fine particles in the North Yellow Sea [D]. Institute of Oceanography, Chinese Academy of Sciences, 2000. 
9 He Qixiang, Li Shaoquan, Liu Jian. Classification of marine clastic sediments [J]. Marine Geology and Quaternary Geology, 2002,22 (001): 115-121.

10 Zhang Fuyuan, Zhang Weiyan, Zhang Xiaoyu, et al. Key techniques and schemes for classification and nomenclature of deep-sea sediments [J]. Journal of Geosciences China University of Geosciences, 2012,37 (001): 93-104.

11 Liu Fucheng, Peng Jun, Zhang Rui, et al. A nonparametric method for generating offshore sediment type maps [J]. Marine Bulletin, 2011.

12 Lark R M , Dove D, Green S L, et al. Spatial prediction of seabed sediment texture classes by cokriging from a legacy database of point observations[J]. Sedimentary Geology, 2012, 281(DEC.15):35-49. 